\title{
高温超导微波限幅滤波器
}

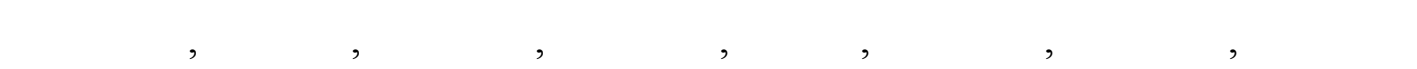

(1) 电子科技大学电子薄膜与集成器件国家重点实验室, 成都 610054 ;

(2) 电子科技大学空天技术研究院, 成都 610054 ;

(3) 南京电子技术研究所, 南京 210013

E-mail: bsr@uestc.edu.cn

2007-12-29 收稿, 2008-04-16 接受

国家高技术研究发展计划(编号: 2007AA03Z212)和电子科技大学青年基金(批准号: L08047401JX0649)资助项目

摘要 利用高温超导薄膜材料的临界电流特性, 并与微波谐振器的电流分布特性相结 合, 在同一微波器件中实现限幅和滤波功能. 理论分析和实验结果表明, 在通讯和雷达系统 应用中, 该器件可以在极大提高系统灵敏度的同时增强系统抗干扰和抗烧毁能力.

关键词

高温超导

滤波器

限幅器

接收机

抗干扰
高温超导薄膜材料在微波器件中的应用已进入 产业化阶段, 其典型应用包括移动通信基站接收系 统以及各类军民用通信和雷达接收前端等 ${ }^{[1]}$. 在目 前的高温超导接收系统中, 利用超导薄膜的低微波 损耗特性是应用的重点, 而据此特性设计的微波滤 波器则形成当前产业化应用的主流. 事实上, 高温超 导材料还具有许多独特性能, 如临界电流特性、非线 性特性和量子效应等, 对于这些特性的研究也已形 成各种器件应用, 包括利用临界电流特性进行工作 的微波限幅器 ${ }^{[2]}$ 、利用量子效应工作的各种Josephson 器件等. 但是由于各种特性需要在独特的工艺条件及 电路布局设计下才能达到最佳应用效果, 因此目前的 许多器件应用都处于追求单一器件的最佳性能设计上, 使得超导材料本身的各种优异性能不能同时体现. 如 果需要同时应用多种性能, 目前的解决方法主要是将 不同的超导器件集成在一起使用, 这样的优点很明显, 但也存在工艺复杂、整体性能低等问题.

本文从通信系统接收前端的结构出发, 分析超 导材料引入系统对系统性能及结构的影响, 并据此 提出限幅/滤波合一器件的必要性和可能性. 然后以 超导材料的特性为基础, 结合滤波器结构讨论限幅 滤波器的构建方案, 提出了以滤波器为框架, 在谐振
器内部引入微桥来实现限幅滤波的方案, 并完成了 原型限幅滤波器件的设计、加工和测试. 最后对测试 结果进行了分析.

\section{1 高温超导接收前端}

\section{1 常规接收机前端}

常规微波接收机前端 [3]一般由图 1(a)和(b)所示的 两种结构构成. 该接收机前端包括天线、限幅器、低 噪声放大器、带通滤波器和下变频模块等, 完成微波

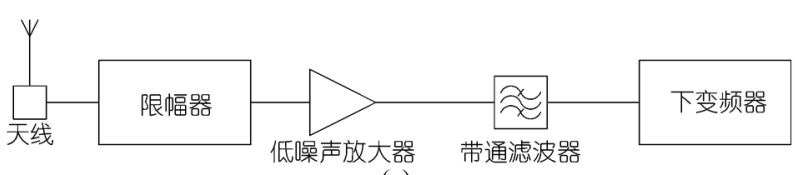

(a)

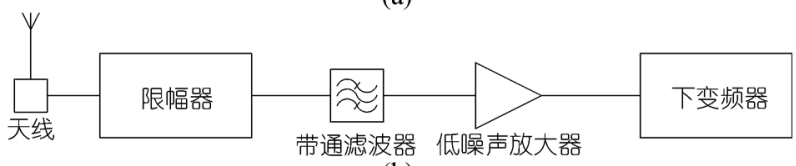

(b)

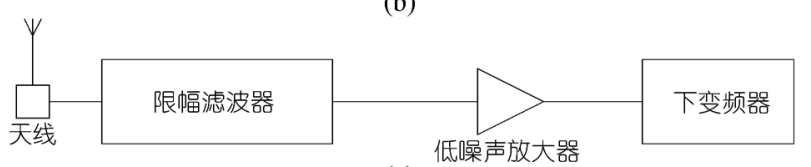

(c)

图 1 微波接收机的构成

(a) 低噪放前置的接收前端; (b) 低噪放后置于滤波器的接收前端;

(c) 高温超导接收前端 
信号的接收、放大和混频处理功能. 为完成信号的高 质量接收, 需要从最佳系统噪声设计考虑, 通常当滤 波器噪声大于低噪放噪声时, 系统设计需要后置滤 波器(图 1(a)), 反之则前置滤波器(图 1(b)). 图中限幅 器主要用于对敏感元件低噪声放大器进行电平保护. 这种传统的系统方案最大的缺点是限幅器在受到通 带外强信号干扰时容易被阻塞, 不能适应现代复杂 电磁环境下的通讯需求.

\section{2 高温超导限幅接收前端}

如果将图 1(b)中的限幅器和带通滤波器做在一 起(图 1(c)), 对于带外强干扰信号就可以由滤波器反 射抑制而不会造成阻塞 ${ }^{[4]}$, 带内则由限幅器进行保护. 这样的接收方案在系统噪声 (一个器件的噪声比两个 器件的噪声低)、抗干扰性能和抗烧毁能力方面将大 大优于传统方案. 而系统的关键就是限幅滤波器的 实现, 就现有文献和专利资料来看, 目前还没有相关 的常规器件具有这样的功能.

\section{2 高温超导限幅滤波器的实现}

对于高温超导微波限幅器, 目前主要有两种方 案实现, 其中一种是通过在均匀微带传输线上加微 桥结构实现, 另外一种是利用共面波导结构实现微 细 $50 \Omega$ 传输线条来达到限幅目的. 对比这两种结构 的微波限幅器, 可以发现微桥结构限幅器对实现工
艺要求不高但适用带宽较窄, 而共面波导结构要求 工艺高但适用带宽非常宽(几乎可以适用于全微波频 段). 对于高温超导微波滤波器, 最常见的结构是由 半波长微带谐振器通过各种耦合方式构成, 现在需 要构成微波限幅滤波器, 最直观的方法就是将限幅 器采用的两种方案直接应用于微带滤波器的谐振器 上, 为此需要对这两种方案进行对比分析.

与高温超导微波限幅器工作的行波状态相比, 微波滤波器中谐振器上的微波电流主要处于驻波状 态, 这种谐振器工作在基波谐振状态时, 谐振器中部 的微波电流分布最强(沿谐振器方向的电流分布). 因 此, 如果要在谐振器内部引入微桥结构实现限幅滤 波器, 则需要选取微桥的位置, 为了达到最大的限幅 效果, 我们考虑在微波电流最强的地方引入微桥.

下面以开口环谐振器滤波器 ${ }^{[5]}$ 为例, 先对比均 匀微带线谐振器滤波器(如图 2(a), 图中谐振器上谐 振电流越强, 灰度级越高, 下同) 和在谐振器内部引 入微桥的滤波器(如图 2(b), 其中微桥宽度为 $10 \mu \mathrm{m}$, 长度为 $200 \mu \mathrm{m}$ )两者的谐振电流分布情况. 如图 2(a) 所示, 均匀微带线谐振器上的电流分布沿谐振器方 向呈抛物线分布, 两端电流小, 中间电流大. 而对于 引入微桥的谐振器, 谐振时谐振电流大大集中在微桥 上(见图 2(b) (d)). 由于微桥上集中的微波电流对限幅 很有利, 同时滤波器工作带宽不宽而且由于长细

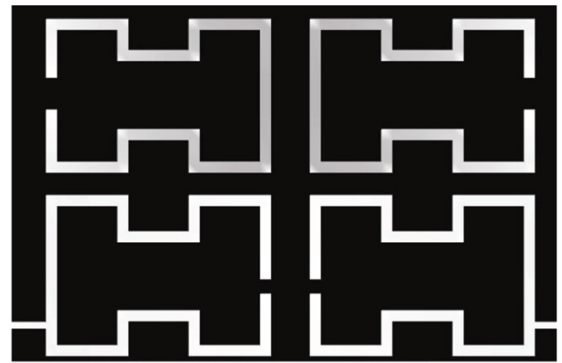

(a)

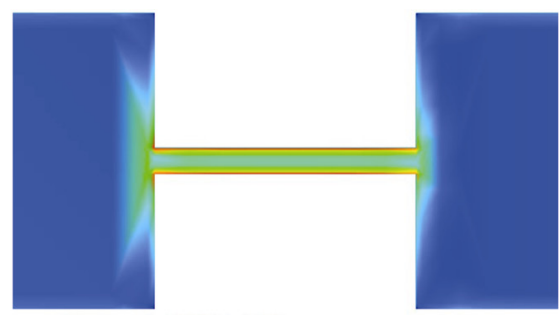

(c)

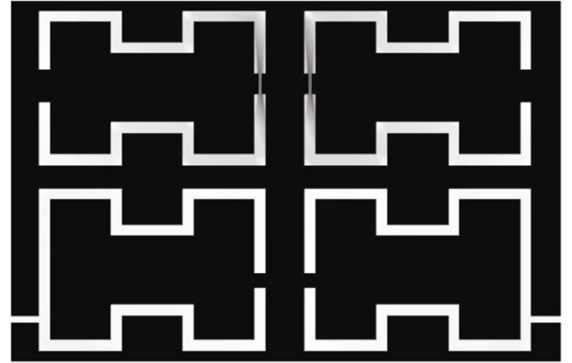

(b)

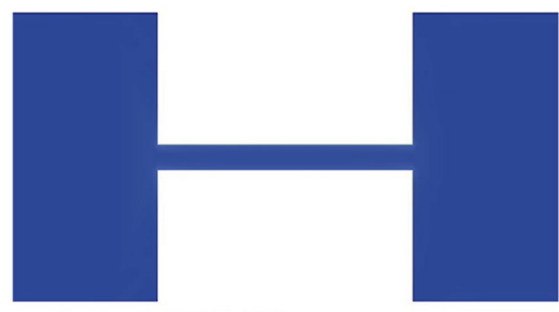

(d)

图 2 微桥结构引入对滤波器谐振电流分布的影响

(a) 均匀线开口环滤波器的谐振电流分布; (b) 谐振器内部引入微桥的开口环滤波器的谐振电流分布; (c) 谐振时微桥部分电流分布仿真 结果(谐振频率处, 红色电流分布强, 蓝色电流分布弱); (d) 非谐振时微桥部分电流分布仿真结果 
微带线滤波器难以实现, 我们可以选定微桥结构的 限幅滤波器实现.

\section{3 限幅滤波器的制作及测试结果}

按上述思路我们在 $\mathrm{YBCO} / \mathrm{LAO} / \mathrm{YBCO}$ 双面高温 超导薄膜 ${ }^{[6]}$ 上设计并制作了工作在 $\mathrm{S}$ 波段的高温超导 限幅滤波器. 该滤波器采用标准湿法光刻工艺完成. 图 3 是在输入功率为 $-10 \mathrm{dBm}$ 时测得的滤波器性能, 图 4 是在输入功率为 $15 \mathrm{dBm}$ 时测得的滤波器性能. 滤波器的限幅功率在滤波器中心频率按文献[7]所述 方案进行测试, 测试结果如图 4 所示. 该滤波器针对 接收机系统应用的结果及系统应用情况见文献[4].

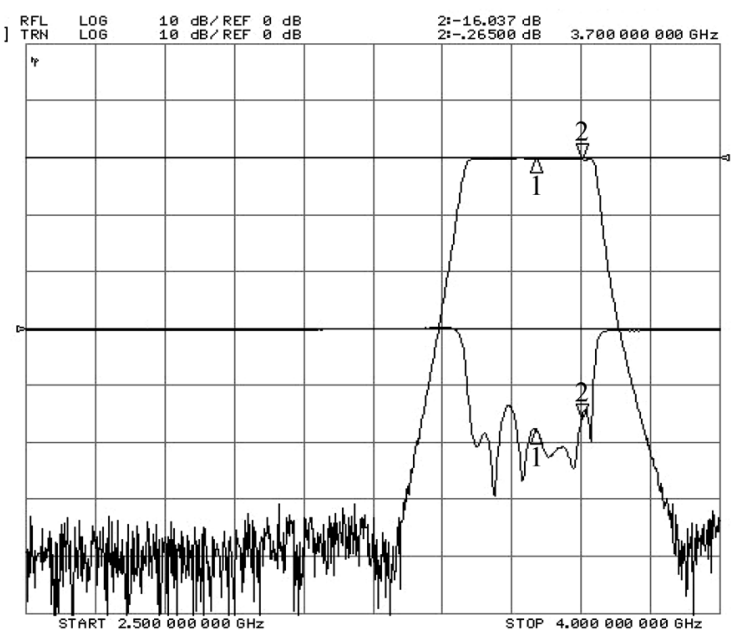

图 $3 \mathrm{~S}$ 波段限幅滤波器低功率情况下的滤波特性 输入功率为 $-10 \mathrm{dBm}$

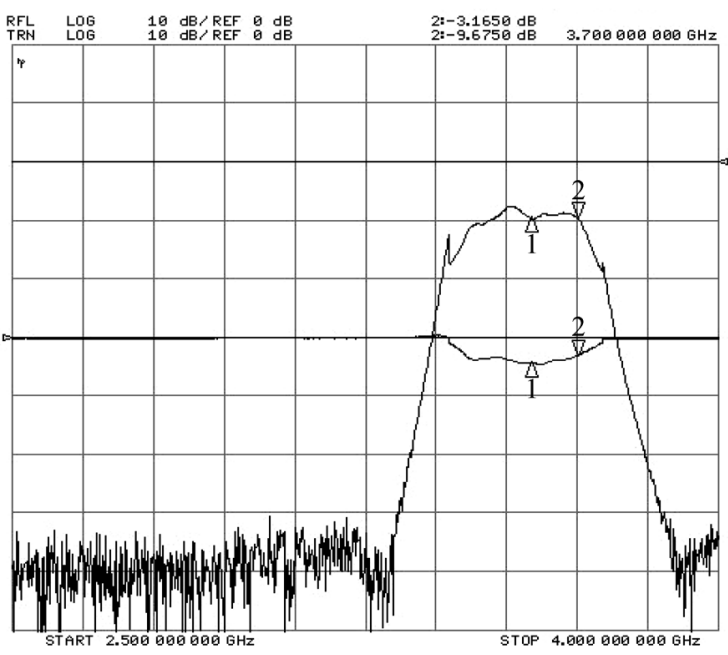

图 $4 \mathrm{~S}$ 波段限幅滤波器限幅情况下的滤波特性 输入功率为 $15 \mathrm{dBm}$

\section{4 测试结果分析}

从图 3 的滤波器测试结果来看, 整个通带内的插 入损耗小于 $0.3 \mathrm{~dB}$, 略大于普通微带线高温超导滤波 器 [8], 这是由于谐振器内部引入微桥结构造成谐振 器 $Q$ 值下降, 从滤波器的反射、形状和带外抑制来看, 微桥结构的引入并无明显影响.

图 4 是输入功率超过限幅电平时的滤波器测试 形状. 从图中可以看到, 当输入功率超过限幅电平时 滤波器起衰减器作用, 但由于谐振器是频率敏感元件, 整体衰减性能并不平坦, 若能实现平坦衰减或对于窄 带信号, 该限幅滤波器即使限幅工作后也可以在后端 检测信号, 将可起到自动增益调整器的作用, 这一特 点可能是该器件与常规限幅器相比的最大优势.

从图 5 所示的功率测试结果可以看到，本文设 计的限幅滤波器限幅电平为 $12.5 \mathrm{dBm}$, 该限幅电平 与常规零偏压 PIN 二极管限幅器限幅电平相当, 但 该限幅电平是在微带微桥线宽为 $10 \mu \mathrm{m}$ 时获得的, 如果选用更窄的微桥线宽将可以获得更低的限幅电 平，显然该限幅器与常规 PIN 二极管相比具有更大 的限幅电平设计灵活性, 并且不需要复杂的偏压电 路来实现.

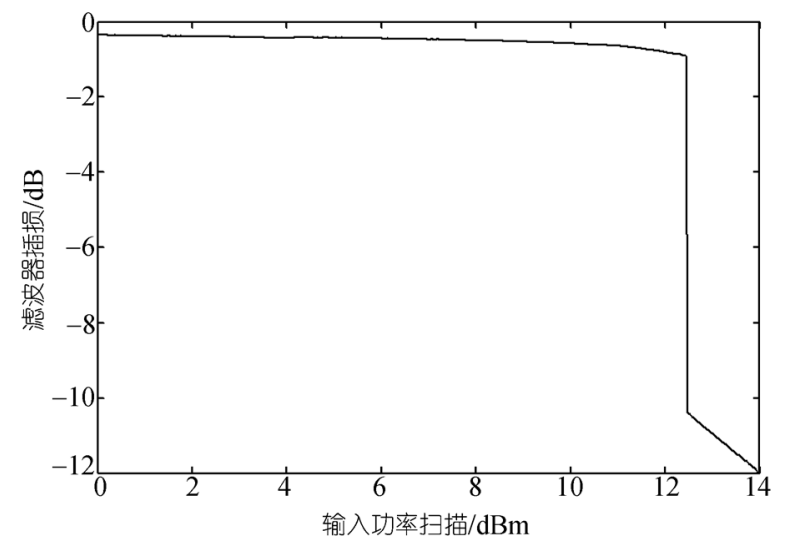

图 5 限幅滤波器功率容量测试结果

对于接收系统使用该限幅滤波器的效果详见文 献[4]，在灵敏度、抗干扰性能和抗烧毁能力等方面均 大大优于常规接收系统, 并在抗干扰性能和抗烧毁 能力方面优于普通高温超导接收机前端系统.

\section{5 结论}

本文同时利用高温超导薄膜的低微波损耗特性 和临界电流特性构建并实现了高温超导限幅滤波器, 
单一器件测试和系统测试证明该方案可行. 该器件 的成功实现证明, 同时利用高温超导材料的多种特 性实现创新型器件将大大拓展高温超导材料的应用
范围, 并体现高温超导材料不可替代的重要性. 关于 高温超导限幅滤波器的研究还需要在限幅电平设计 等与材料科学直接相关的领域作进一步工作.

\section{参考文献}

1 Mansour R R. Microwave superconductivity. IEEE Trans Microw Theory Tech, 2002, 50: 750-759 [DOI]

2 Booth J C, Rudman D A, Ono R H. A self-attenuating superconducting transmission line for use as a microwave power limiter. IEEE Trans Appl Supercond, 2003, 13(2): 305-310 [DOI]

3 Laskar J, Matinpour B, Chakraborty S. Modern Receiver Front-Ends: Systems, Circuits, and Integration. Hoboken, New Jersey: John Wiley \& Sons, Inc. 2004

4 羊恺, 补世荣, 刘娟秀, 等. 限幅自保护高温超导接收机前端研究. 电子科技大学学报, 2007, 36(2): 223一 226

5 Hong J S, Lancaster M J. Couplings of microstrip square open-loop resonators for cross-coupled planar microwave filters. IEEE Trans-MTT, 1996, 44(9): 2099-2109 [DOD]

6 Tao B W, Chen J J, Liu X Z, et al. Speed modulation technique to achieve simultaneous deposition of 3-in. double-sided Y-Ba-Cu-O thin films. Physica C, 2005, 433: 87-92[DOI]

7 Shen Z Y. High-temperature superconducting microwave circuits. Boston, MA: Artech House, 1994

8 羊恺，补世荣，张其劭，等。高温超导小型化多曲折线滤波器研制. 科学通报, 2002, 49(18): 1378-1380 